\title{
Intoxicações por plantas em ruminantes e equídeos na região central de Rondônia
}

\author{
Plant poisonings in ruminants and equidae in central region of Rondônia state, Northern Brazil
}

\author{
Sandro Vargas Schons ${ }^{I}$ Tiago Vaz LopesII Taciane Leticia de MeloII João Padovani Lima ${ }^{I I}$ \\ Franklin Riet-Correa ${ }^{\text {III }}$ Miguel Ângelo de Brito Barros ${ }^{\mathrm{IV}}$ Ana Lucia Pereira Schild ${ }^{\mathrm{V}}$
}

\section{RESUMO}

Foi realizado um levantamento em 12 municípios da região central de Rondônia sobre a presença de plantas tóxicas e ocorrência de surtos de intoxicação em ruminantes $e$ equídeos. O trabalho foi desenvolvido mediante a utilização de um questionário aplicado a médicos veterinários, agrônomos, zootecnistas e produtores rurais, com o objetivo de identificar as principais plantas tóxicas que ocorrem na região. Trinta e quatro entrevistados relataram casos de intoxicação por uma ou mais plantas comprovadamente tóxicas como: Palicourea marcgravii (12 surtos), Palicourea grandiflora e Enterolobium contortisiliquum (sete surtos cada) e Palicourea juruana, Brachiaria radicans, Brachiaria brizantha e Manihot esculenta (dois surtos cada). Em ovinos, foram relatados dois surtos de fotossensiblização por Brachiaria decumbens e um surto de mortalidade por Palicourea grandiflora. Dos 34 surtos relatados em bovinos pelos entrevistados, 374 (8,9\%) animais foram afetados e 311 (7,4\%) morreram, de um total de 4.192 de ambos os sexos sob risco. De um total de 250 ovinos sob risco, três surtos de intoxicação por plantas foram relatados e afetaram 28 animais, dos quais 20 morreram. Amorimia sp., previamente desconhecida como tóxica, foi identificada como causa de morte súbita em $32 \%$ das propriedades. Quinze surtos de cólica em equídeos que pastavam cultivares de Panicum maximum ('Massai', 'Tanzânia'e 'Mombaça') durante o período das chuvas foram, também, observados. Os resultados do presente trabalho demonstram a importância significativa das intoxicações por plantas como causa de perdas econômicas para a pecuária da região central do Estado de Rondônia. Com a realização deste trabalho, o número de plantas tóxicas para ruminantes com a confirmação de ocorrência de surtos com mortalidade na região passou de um para nove, o que confirma que um trabalho sistemático de investigação é necessário para o conhecimento da importância das intoxicações por plantas na região Norte do Brasil.

Palavras-chave: doenças de rebanho, plantas tóxicas, ruminantes, equídeos, Rondônia.

\section{ABSTRACT}

A survey about the presence of toxic plants and the occurrence of outbreaks of poisoning in ruminants and equidae was performed in 12 municipalities of the central region of the state of Rondônia. Ninety eight persons were interviewed, including farmers, veterinary practitioners, agronomists, and agrarian technicians. Thirty four farmers reported poisoning by toxic plants, including poisoning by Palicourea marcgravii (12 outbreaks), Palicourea grandiflora and Enterolobium contortisiliquum (seven outbreaks each), and Palicourea juruana, Brachiaria radicans, Brachiaria brizantha, and Manihot esculenta (two outbreaks each). In sheep, farmers reported two outbreaks of photosensitization caused by Brachiaria decumbens and one outbreak of sudden death caused by Palicourea grandiflora. In the 34 outbreaks, 374 (8,9\%) bovines were affected and 311 (7.4\%) died, from a total of 4.192 cattle exposed. In the three outbreaks in sheep, 28 animals were affected and 20 died out of 250 exposed. Amorimia sp., previously misidentified as Mascagnia sepium, a previously unreported toxic plant, was identified as a cause

IPrograma de Pós-graduação em Veterinária, Faculdade de Veterinária (FV), Universidade Federal de Pelotas (UFPel), Campus Universitário, s/n, Pelotas, RS, Brasil.

IICurso de Medicina Veterinária, Centro Universitário Luterano de Ji-paraná (CULJI), Universidade Luterana do Brasil (ULBRA), Ji-Paraná, RO, Brasil.

IIHospital Veterinário, Centro de Saúde e Tecnologia Rural (CSTR), Universidade Federal de Campina Grande (UFCG), Patos, PB, Brasil.

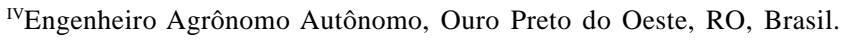

Laboratório Regional de Diagnóstico (LRD), FV, UFPel, Campus Universitário, s/n, 96010-900, Pelotas, RS, Brasil. E-mail: alschild@terra.com.br. Autor para correspondência. 
of sudden death in sheep and cattle in $32 \%$ of the farms. Fifteen outbreaks of colic in horses grazing Panicum maximum (cultivars 'Massai', 'Tanzânia', and 'Mombaça') during the rainy season were also reported. It is concluded that poisoning by toxic plants is an important cause of economic losses in livestock in the region studied. With the results of this research the number of known toxic plant for ruminants in central region of Rondônia increased from one to nine, indicating that more research is necessary for the knowledge of poisonous plants for livestock in the Brazilian Amazonic region.

Key words: Livestock disease, poisonous plants, ruminants, equidae, Rondônia.

\section{INTRODUÇÃO}

As criações de animais nos estados do Norte apresentam características singulares em relação às demais regiões do Brasil. Primeiramente, chama atenção a estreita relação dos animais com áreas de florestas abertas, muitas vezes, substituídas por pastagens cultivadas. Essas florestas são mundialmente conhecidas pela grande biodiversidade ainda pouco estudada (OLIVEIRA, 2009). O desconhecimento e a falta de profissionais especializados no Estado de Rondônia têm gerado elevados prejuízos à produção animal, principalmente com relação à mortalidade dos animais por causas desconhecidas. Produtores rurais e médicos veterinários que atuam na região consideram, como principal causa de mortalidade em animais de produção, a escassez de alimentos durante a época de estiagem e a ingestão de plantas tóxicas.

Quatorze espécies de plantas comprovadamente tóxicas são responsáveis por mortalidade de animais de produção nos estados do norte do país (TOKARNIA et al., 2007). Dentre essas espécies, destacam-se Palicourea marcgravii, $\boldsymbol{P}$. juruana e $\boldsymbol{P}$. grandiflora que provocam surtos de morte súbita e grandes perdas econômicas, principalmente nos estados do Amazonas, norte do Mato Grosso, norte de Goiás, Roraima, Amapá, Rondônia, Acre e oeste do Maranhão. São identificadas, ainda, outras plantas como Arrabideae bilabiata e A. japurensis, Ipomoea fistulosa e I. asarifolia, Lantana spp., Ricinus communis, Manihot spp., Pteridium aquilinum e Brachiaria spp. (TOKARNIA et al., 2007). Devido à carência de dados sobre as causas de mortalidade em animais por tais plantas, fica difícil de estimar as perdas econômicas causadas por elas no estado de Rondônia, onde apenas uma planta, a Palicourea grandiflora havia sido identificada como causa de mortalidade em bovinos (TOKARNIA et al., 2007), embora outras espécies do gênero reconhecidamente tóxicas como $\boldsymbol{P}$. marcgravii e $\boldsymbol{P}$. juruana também sejam encontradas no Estado (TOKARNIA et al., 2000).
O estudo sistemático das plantas tóxicas em regiões com poucas pesquisas sobre elas aumenta consideravelmente o número de espécies tóxicas conhecidas (SILVA et al., 2006). Um exemplo disso é o estado da Paraíba, onde até o ano de 2000 eram conhecidas oito plantas tóxicas e esse número aumentou para 21 após iniciar-se um estudo sobre o assunto neste Estado (RIET-CORREA et al., 2006).

Considerando a falta de informações sobre o significativo impacto das intoxicações por plantas em animais de produção na Região Central de Rondônia, este trabalho teve por objetivo identificar as plantas tóxicas que ocorrem na região, sugeridas e/ou confirmadas como causadoras de mortalidade de bovinos e equídeos, estabelecendo os aspectos epidemiológicos, bem como os achados clínicopatológicos envolvidos nessas intoxicações.

\section{MATERIAL E MÉTODOS}

Para identificação das principais plantas tóxicas conhecidas pelos produtores rurais da Região Central de Rondônia, foram realizadas visitas técnicas a 98 propriedades de 12 municípios : Ji-paraná, Cacoal, Ouro Preto do Oeste, Presidente Médice, Vale do Anari, Rolim de Moura, Seringueiras, Monte Negro, Alta Floresta, Colorado do Oeste, Costa Marques e Nova União (Figura 1), no período de 2008 a 2011. Durante as visitas, foram feitas entrevistas a médicos veterinários, agrônomos, zootecnistas e produtores rurais. A entrevista foi baseada no preenchimento de três questionários semelhantes ao utilizados por SILVA et al. (2006), sendo entrevistadas pelo menos três pessoas por município.

Os dados coletados nas entrevistas foram sistematizados e foi realizado o acompanhamento, quando possível, dos surtos de mortalidade de animais de produção em que o quadro epidemiológico e clínico sugeria intoxicação por plantas. O diagnóstico foi realizado pela epidemiologia e sinais clínicos e, quando possível, também pela patologia macroscópica e histológica. Amostras das plantas suspeitas foram coletadas para identificação.

\section{RESULTADOS}

Durante as visitas às propriedades, foram identificadas 16 plantas mencionadas pelos produtores e reconhecidas na literatura como tóxicas, estabelecendo-se o percentual de propriedades em que elas eram encontradas: Palicourea marcgravii (80\%), Asclepias curassavica (79\%), Palicourea grandiflora (67\%), Brachiaria sp. (66\%), Enterolobium

Ciência Rural, v.42, n.7, jul, 2012. 


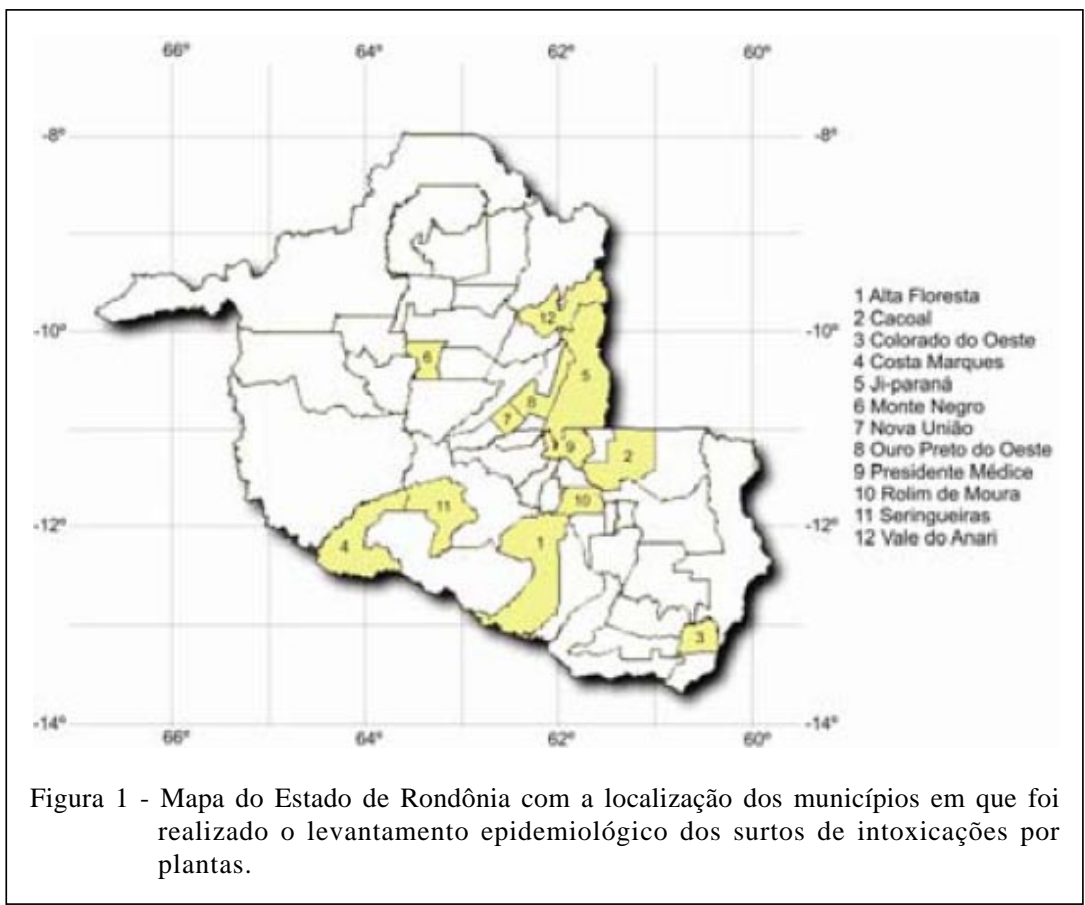

contortisiliquum (65\%), Pteridium aquilinum (57\%), Brachiaria radicans (50\%), Lantana camara (47\%), Senna occidentalis (40\%), Ricinus communis (33\%), Manihot esculenta (33\%), Ipomea carnea (20\%), Ipomoea asarifolia (17\%), Palicourea juruana (17\%), Crotalaria spp. (16\%) e Arrabidaea bilabiata (6\%).

Além das plantas conhecidas, os produtores relataram, também, a presença de três plantas suspeitas de serem tóxicas e causarem mortes em animais na propriedade, das quais apenas Amorimia sp., conhecida como "tingui" e presente em 32\% das propriedades, foi confirmada como tóxica. Anteriormente, essa planta tinha sido identificada, erroneamente, como Mascagnia sepium (SCHONS et al., 2011).

Trinta e quatro produtores relataram casos sugestivos de intoxicação em bovinos e ovinos por uma ou mais plantas. Foram relatados, também, casos de cólica em equídeos que pastavam variedades de Panicum maximum (Massai, Tanzânia e Mombaça) durante o período das chuvas.

Casos de intoxicação em bovinos por Palicourea marcgravii, $\boldsymbol{P}$. grandiflora e $\boldsymbol{P}$. juruana foram relatados em 21 propriedades da Região Central de Rondônia. $\boldsymbol{P}$. marcgravii foi responsável pela maioria dos relatos (57\%), seguido da $\boldsymbol{P}$. grandiflora (33\%) e $\boldsymbol{P}$. juruana (9,5\%). De um total de 3.549 bovinos, 496 apresentaram sinais clínicos sugestivos da intoxicação pelas diferentes espécies de Palicourea e 253 morreram após 4 a 12 minutos. A morbidade observada nestes casos foi de $14 \%$ a $16 \%$ e a mortalidade de $4 \%$ a $16 \%$. Não foi observada predileção por sexo, raça e idade, embora os bovinos de 12 a 24 meses tenham sido os mais acometidos (50\% dos casos). As intoxicações foram observadas em todas as épocas do ano, mas, no período do início das chuvas, de outubro a dezembro, a ocorrência foi maior (54\%) e menor no período chuvoso de fevereiro a abril (9\%). Em ovinos, somente um proprietário relatou ter observado casos sugestivos de intoxicação por $\boldsymbol{P}$. grandiflora. Esse surto ocorreu no município de Monte Negro, durante o período da seca. O surto ocorreu em um lote de 200 ovinos com idade de 8 a 12 meses que estavam sendo trocados de piquete e apresentaram sinais clínicos de tremores musculares, queda lateral e morte em alguns minutos.

Surtos de morte súbita em bovinos e ovinos ocorreram em consequência da ingestão de Amorimia sp., durante a estação chuvosa no Vale do Anari, após a movimentação dos animais. Em um dos surtos relatados, morreram 14 ovinos de um total de 35, sem observação de sinais clínicos. O fato ocorreu 10 horas após os animais serem colocados em uma área onde o proprietário havia cortado plantas de tingui (Amorimia sp.) e deixado para secar. Em outra propriedade, de 28 novilhas adquiridas e colocadas em potreiro com a planta, sete morreram sem terem sido movimentadas ou excitadas. Os demais bovinos foram retirados da área e mais três morreram durante a transferência. Em 
outra propriedade, foram separados oito bovinos para a venda e morreram sete após serem movimentados. Em uma terceira propriedade, 200 bovinos foram movimentados para vacina e morreram 10 no interior do curral. Neste caso, como a planta não estava no grupo das plantas comprovadamente tóxicas, foram realizados experimentos em ovinos e confirmada sua toxicidade (SCHONS et al., 2011).

Em duas propriedades localizadas nos municípios de Cacoal e Ji-paraná, respectivamente, foram observados bovinos com lesões de fotossensibilização hepatógena sugestivos de intoxicação por Brachiaria brizantha, no início das chuvas, nos meses de novembro e dezembro de 2009. No município de Cacoal, os casos foram observados em cinco novilhas de um lote de 35 animais. Na propriedade localizada no município de Ji-paraná, os casos ocorreram em 12 bezerros com idades entre 8 e 12 meses, que estavam sendo desmamados. Fotossensibilização hepatógena em ovinos, causada provavelmente pela ingestão de B. decumbens (DRIEMEIER et al., 2002; SATURNINO et al., 2010), foi observada em duas propriedades localizadas no município de Ji-paraná. O primeiro caso foi observado em um lote de 20 ovinos confinados, de 6 a 8 meses, desmamados, os quais foram colocados em uma pastagem da gramínea. O segundo relato ocorreu em ovinos criados extensivamente em pastagem de $\boldsymbol{B}$. decumbens.

Em sete propriedades localizadas nos municípios de Ji-paraná, Ouro Preto do Oeste e
Presidente Médice, foram relatados casos de intoxicação por Enterolobium contortisiliquum em bovinos durante os meses de outubro e novembro, época marcada por elevada carência de alimentos e queda das favas. Conforme os produtores, casos de mortalidade e lesões de fotossensibilização foram frequentemente observados em anos anteriores. Nos surtos observados, a morbidade foi de $41 \%$ e a mortalidade de $18 \%$. Em todos os casos, foram descritos sinais clínicos de emagrecimento acentuado, desidratação e diarreia verde-escura e fétida. Casos com fotossensibilização (Figura 2) foram relatados em cinco surtos. Em quatro surtos, na mesma época, ocorreram abortos sugestivos da intoxicação pelas favas. Os fetos abortados apresentavam autólise acentuada dificultando a avaliação macroscópica e histopatológica. Dois bovinos necropsiados em um surto acompanhado em Ji-Paraná apresentavam lesões caracterizadas por fígado amarelado ou alaranjado e aumentado de tamanho, distensão da vesícula biliar e presença de favas de $\boldsymbol{E}$. contortisiliquum no rúmen e retículo. Histologicamente, havia vacuolização fina dos hepatócitos e enterite linfocítica moderada.

Surtos de intoxicação em bovinos, causados provavelmente por Brachiaria radicans, conhecida na região como bico-de-pato, bambuzinho, braquiária do brejo e Tanner grass foram relatados em três propriedades dos municípios de Ji-paraná, Seringueiras e Presidente Médice. O surto relatado no município de Presidente Médice ocorreu em outubro, correspondendo ao período de transição entre as

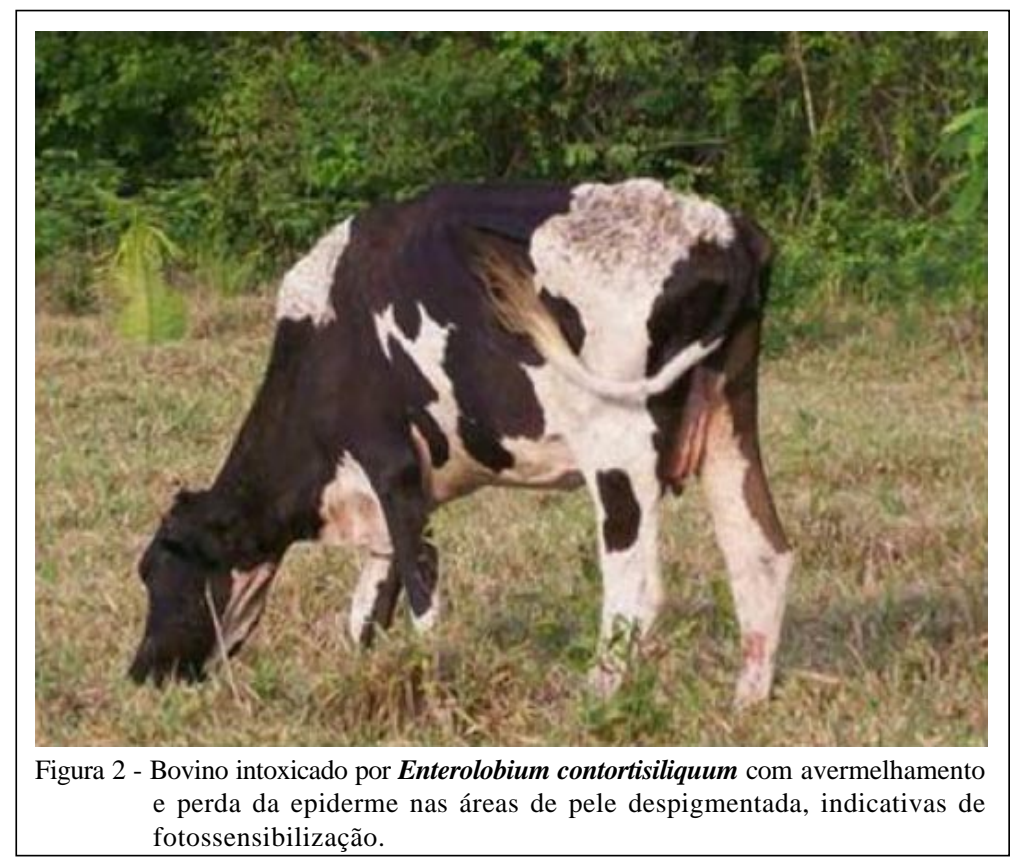

Ciência Rural, v.42, n.7, jul, 2012. 
estações seca e chuvosa (BEZERRA et al., 2010). Nove bovinos com idade superior a dois anos apresentaram emagrecimento, andar cambaleante, anemia e hemoglobinúria, com curso clínico de 3-5 dias. Seis bovinos intoxicados morreram e três recuperam-se 10 dias após serem retirados das pastagens de $\boldsymbol{B}$. radicans. Os proprietários relataram que, durante o período da seca, os animais eram trocados para potreiros que apresentavam solo úmido, com material orgânico em decomposição e com $\boldsymbol{B}$. radicans em crescimento.

Intoxicação por Manihot esculenta foi relatada em duas propriedades localizadas nos municípios de Vale do Anari e Alta Floresta. No primeiro surto, os três bovinos foram intoxicados quando beberam a água utilizada para lavar as raízes quebradas de $\boldsymbol{M}$. esculenta durante a fabricação de farinha. O segundo surto de intoxicação, no município de Alta Floresta, ocorreu em um lote de 200 bovinos que estavam recebendo mandioca triturada ensilada no cocho. Após a ingestão deste alimento, foram observados quatro animais com dificuldade respiratória, tremores musculares e decúbito lateral. Após seis horas, três bovinos recuperam-se e um morreu.

Durante o período do estudo, ocorreram 15 surtos com manifestação clínica de cólica em equinos mantidos em pastagens de Panicum maximum nas variedades Massai, Tanzânia e Mombaça, em diversos municípios da região central de Rondônia. De um total de 103 equídeos, de ambos os sexos e de várias idades e raças, 44 (42\%) apresentaram sinais clínicos de cólica (Figura 3A e B) e 31 (30,1\%) e morreram após curso clínico de 9-72 horas. Todos os surtos ocorreram após os animais serem introduzidos nas pastagens de Panicum durante os meses de novembro a abril, que corresponde à época das chuvas na região. Animais mantidos durante o ano todo na gramínea não apresentaram sinais da intoxicação, somente quando retirados e recolocados na pastagem, após 3 ou 4 dias. Duas propriedades tiveram casos de cólica após a roçada da gramínea. Foram necropsiados 12 equídeos, provenientes dos surtos e as lesões macroscópicas observadas caracterizaram-se por distensão das paredes do estômago, alças intestinais e ceco, devido ao acúmulo de gás (Figura 3C). O estômago apresentava hemorragia na mucosa da porção glandular (Figura 3D) e conteúdo líquido. O duodeno, jejuno, íleo e ceco apresentavam conteúdo líquido, com exceção do ceco, que apresentava conteúdo compactado e a mucosa hemorrágica. As lesões histológicas no sistema digestivo dos equinos necropsiados caracterizavamse por congestão e hemorragias na mucosa, especialmente do estômago e intestino delgado. Havia também gastrite e enterite difusa com presença de células inflamatórias mononucleares na lâmina própria desses órgãos. O diagnóstico neste caso foi realizado

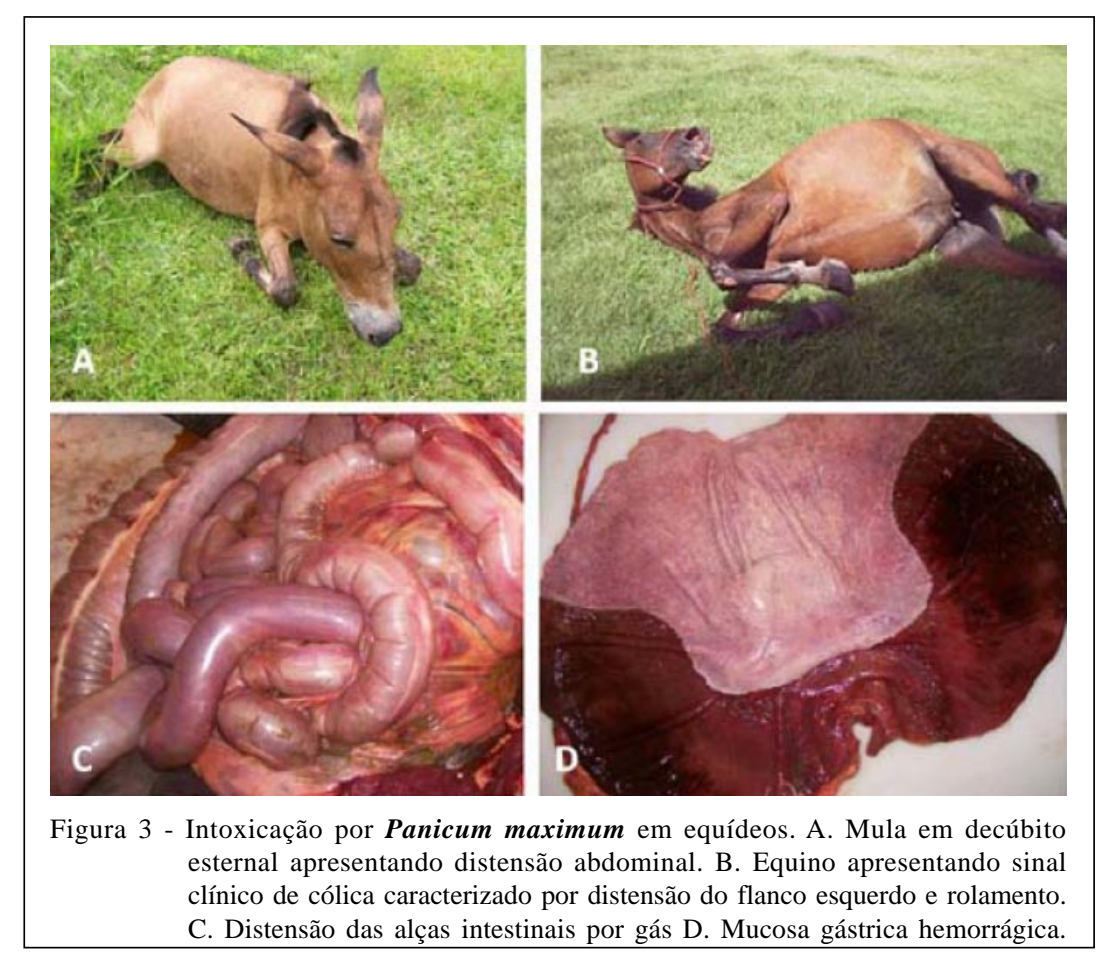

Ciência Rural, v.42, n.7, jul, 2012. 
pelos sinais clínicos, alterações anatomohistopatologicas e confirmado pela reprodução experimental da intoxicação. Foram administrados a 11 equídeos as variedades Mombaça, Tanzânia e Massai de $\boldsymbol{P}$. maximum nas diferentes fases de crescimento e reproduzido um quadro de cólica com lesões macroscópicas e histológicas similares às observadas nos casos espontâneos (SCHONS, 2011. Dados não publicados).

\section{DISCUSSÃO}

Os resultados do presente trabalho demonstraram a ocorrência de 10 diferentes plantas tóxicas causando surtos de mortalidade na região central do estado de Rondônia em ruminantes e equídeos. Até então, apenas $\boldsymbol{P}$. grandiflora havia sido descrita como causa de morte de animais de produção neste Estado (TOKARNIA et al., 1981; 2007). Foi observado que, em 34 surtos de intoxicações por plantas, relatados em bovinos em 12 municípios da região central de Rondônia, a mortalidade média foi de 7,4\%. Apesar de que os surtos de intoxicação por Palicourea spp., Brachiaria brizantha, Brachiaria decumbens, Brachiaria radicans e Manihot spp. não tenham sido confirmados por necropsia e/ou histopatologia, as evidências epidemiológicas e clínicas associadas à presença dessas plantas sugerem fortemente que os surtos tenham sido causados por elas.

As diferentes espécies de Palicourea foram as plantas mais frequentemente relatadas como presentes nas propriedades e as responsáveis pelo maior número de surtos (21) e percentual de mortes (81,35\%). Estes dados corroboram a afirmação de que o gênero Palicourea é o mais importante como causa de mortalidade em bovinos na região Norte do Brasil, sendo responsável por $80 \%$ das mortes de bovinos causadas por plantas (TOKARNIA et al., 2000; 2007).

Chama a atenção o relato de um surto sugestivo de intoxicação por Palicourea grandiflora em ovinos. Surtos espontâneos de intoxicação pelo gênero Palicourea não têm sido descritos nesta espécie animal, porém a intoxicação por $\boldsymbol{P}$. marcgravii foi reproduzida experimentalmente em ovinos (TOKARNIA et al., 1986). A única planta que causa morte súbita encontrada na propriedade onde a intoxicação ocorreu espontaneamente foi $\boldsymbol{P}$. grandiflora, ficando evidenciado que, apesar de não ser frequente, pode ocorrer em ovinos que tenham acesso a locais onde a planta está presente. Foi mencionado que a intoxicação não ocorre em ovinos ou é rara, devido aos hábitos de alimentação
(TOKARNIA et al., 1986). É provável que, no caso descrito neste trabalho, os ovinos, ao procurarem proteção nas horas de sol mais forte, tenham invadido áreas de capoeira e matas onde a planta ocorre.

As mortes súbitas que ocorriam no Estado de Rondônia eram frequentemente atribuídas às espécies de Palicourea. No entanto, na região central do Estado, a intoxicação por Amorimia sp. deve ser considerada nos casos de ocorrência de morte dessa natureza, tanto em bovinos como em ovinos. Nesta espécie, a intoxicação foi comprovada experimentalmente (SCHONS et al., 2011).

Surtos de fotossensibilização foram observados na região, causados tanto por Brachiaria spp. como por Enterolobium contortisiliquum. No caso deste último, foi observado que a doença ocorre no final da seca, quando há disponibilidade das favas no solo e o pasto está seco. Os mesmos animais apresentaram também sinais clínicos digestivos da intoxicação. Nesses casos, o diagnóstico diferencial epidemiológico é feito pela presença de uma das plantas e pela época do ano, já que a intoxicação por $\boldsymbol{E}$. contortisiliquum é estacional de setembro a novembro, quando as favas estão maduras e caem ao solo (MELLO et al., 2010). Por outro lado, a intoxicação por Brachiaria afeta principalmente animais jovens e que entraram em contato com a gramínea pela primeira vez (SATURNINO et al., 2010, RIET-CORREA et al., 2011).

Os surtos de intoxicação por $\boldsymbol{B}$. radicans ocorreram no início do período chuvoso em áreas que permaneciam úmidas durante a seca e nas quais havia a gramínea em brotação. Nas encostas dos córregos, a planta estava viçosa. A intoxicação por esta gramínea foi descrita no litoral de Santa Catarina (GAVA et al., 2010) e no estado de São Paulo (ROSENFELD et al., 1971), sendo mencionado que a planta se desenvolve bem em solos úmidos (GAVA et al., 2010), do mesmo modo que foi observado nos surtos aqui relatados.

Um dos surtos de intoxicação por $\boldsymbol{M}$. esculenta ocorreu quando bovinos ingeriram a água de lavagem do tubérculo durante a fabricação da farinha. Essa forma de intoxicação não é frequente, uma vez que a maioria dos produtores sabe que o tubérculo é tóxico se ingerido in natura. Esta forma foi mencionada na intoxicação experimental por maniçoba em bovinos (CANELLA et al., 1968). No outro caso, aparentemente, a intoxicação aconteceu devido à presença de alguns tubérculos inteiros na silagem que não perderam a toxicidade. A silagem de M. esculenta é frequentemente utilizada para alimentar os animais em exposições e feiras sem causar intoxicação.

A cólica dos equídeos que pastoreiam diferentes variedades de $\boldsymbol{P}$. maximum tem sido descrita, 
também, no Pará, Acre e Maranhão (CERQUEIRA et al., 2009) com epidemiologia, sinais clínicos e patologia similares aos observados nos casos descritos neste trabalho. Não tem sido determinada a causa dos casos de cólica, porém é sugerido que a presença de carboidratos hidrosolúveis e carboidratos rapidamente fermentáveis seriam a causa da doença (CERQUEIRA et al., 2009).

Verificou-se que Amorimia sp., que ainda não havia sido identificada como tóxica, é uma importante causa de morte súbita em ovinos.

Os resultados do presente trabalho demonstram que várias plantas tóxicas ocorrem na região central do Estado de Rondônia causando surtos de mortalidade em ruminantes e equídeos. Deve ser destacado que, embora alguns relatos de mortalidade associados à ingestão de plantas tóxicas não tenham sido confirmados pela patologia, evidências epidemiológicas e clínicas apontam fortemente para essa etiologia. Dessa forma, o número de plantas tóxicas com a confirmação e/ou suspeita de causarem mortalidade de bovinos e equídeos na região passou de um para nove, o que confirma que um trabalho sistemático de investigação nesta área é necessário para o real conhecimento das principais plantas tóxicas economicamente importantes na região.

\section{AGRADECIMENTOS}

Trabalho financiado pelo INCT para o controle das intoxicações por plantas/CNPq (Proc. nº573534/2008-0).

\section{REFERÊNCIAS}

BEZERRA. R.B. et al. Caracterização temporal da precipitação pluvial do município de Porto Velho/RO no período de 1945 2003. Sociedade \& Natureza v.22 p.609-623, 2010.

CANELLA, C.F.C. et al. Intoxicação experimental pela maniçoba (Manihot glaziovi Muell. Arg.) em bovinos. Pesquisa Agropecuária Brasileira v.3, p.347-350, 1968.

CERQUEIRA, V.D. et al. Colic caused by Panicum maximum toxicosis in equidae in northern Brazil. Journal Veterinary Diagnostic Investigation, v.21, p.882-888, 2009.
DRIEMEIER, D. et al. Study of experimentally induced lesions in sheep by grazing Brachiaria decumbens. Toxicon v.40, p.1027-1031, 2002.

GAVA, A. et al.b Intoxicação espontânea e experimental por Brachiaria radicans (tanner-grass) em bovinos. Pesquisa Veterinária Brasileira, v.30, p.255-259, 2010.

MELLO, G.W.S. et al. Plantas tóxicas para ruminantes e equídeos no Norte Piauiense. Pesquisa Veterinária Brasileira, v.30, p.1-9, 2010

OLIVEIRA, O.A. História, desenvolvimento e colonização do estado de Rondônia. 7.ed. Porto Velho: Rondônia, 2009. 102 p.

RIET-CORREA, F. et al.b Plantas tóxicas da Paraíba. João Pessoa: SEBRAE, 2006. 54p.

RIET-CORREA, B. et al. Intoxicação por Brachiaria spp. em ruminantes no Brasil. Pesquisa Veterinária Brasileira v.31, p.183-192, 2011.

ROSENFELD, G. et al. Anemia hemolítica em bovinos alimentados com Brachiaria sp. (Tanner Grass). Arquivos do Instituto Biológico, v.38, p.267-273, 1971.

SATURNINO, K.C. et al. Intoxicação experimental por Brachiaria decumbens em ovinos confinados. Pesquisa Veterinária Brasileira, v.30, p.195-202, 2010.

SCHONS, S.V. et al. Poisoning by Amorimia (Mascagnia) sepium in sheep in northern Brazil. Toxicon v. 57, p. 781-786, 2011.

SILVA, D.M. et al. Plantas tóxicas para ruminantes e eqüídeos no Seridó Ocidental e Oriental no Rio Grande do Norte. Pesquisa Veterinária Brasileira v. 26, p. 223-236, 2006.

TOKARNiA, C.H. et al. Plantas tóxicas da Amazônia a bovinos e outros herbívoros. Manaus: INPA, 2007. 98 p.

TOKARNIA, C.H. et al. Plantas tóxicas do Brasil. Rio de Janeiro: Editora Helianthus, 2000. 310p.

TOKARNIA, C. H. et al. Intoxicação experimental por Palicourea marcgravii (Rubiacaea) em ovinos. Pesquisa Veterinária Brasileira v.6, p. 121-131, 1986.

TOKARNIA, C.H. et al. Intoxicação por Palicourea grandiflora (Rubiaceae) em bovinos no território de Rondônia. Pesquisa Veterinária Brasileira v. 1, p.85-94, 1981. 